\title{
A la recherche de Claudine ou le libertinage démoniaque
}

\section{Natalia Boyarskaya}

Traducteur : Arnaud Nicod-Clément

\section{OpenEdition \\ Journals}

Édition électronique

URL : http://journals.openedition.org/edl/306

DOI : $10.4000 /$ edl.306

ISSN : 2296-5084

\section{Éditeur}

Université de Lausanne

\section{Édition imprimée}

Date de publication : 15 décembre 2009

Pagination : $39-54$

ISBN : 978-2-940331-21-5

ISSN : 0014-2026

\section{Référence électronique}

Natalia Boyarskaya, «A la recherche de Claudine ou le libertinage démoniaque », Études de lettres [En ligne], 4 | 2009, mis en ligne le 15 décembre 2012, consulté le 19 décembre 2020. URL : http:// journals.openedition.org/edl/306 ; DOI : https://doi.org/10.4000/edl.306 


\section{À LA RECHERCHE DE CLAUDINE OU LE LIBERTINAGE DÉMONIAQUE}

Dans un épisode du roman Le Maître et Marguerite de M. Bulgakov, Marguerite est mise en parallèle avec une certaine Claudine, "veuve infatigable». Nos recherches des origines de cette allusion historique ou littéraire (sont évoqués sous ce rapport plusieurs romans de Colette et une pièce de Molière) apportent une nouvelle façon de lire ce célèbre roman: à la lumière de la tradition dite libertine.

L'idée de cet article est apparue pendant notre travail dans le cadre du projet Libertinage et dandysme dans la culture russe: stratégies de la liberté $^{1}$, à partir d'une question qui tourmente depuis plusieurs années déjà l'auteur de ces lignes: qui est la Claudine mentionnée dans le roman de Mixail Afanas'evič Bulgakov Le Maître et Marguerite [Master i Margarita] (1928-1940)?

Les lecteurs du roman se souviennent que Marguerite, native de Moscou, possède une part importante de sang royal français et est l'«arrière-arrière-arrière-arrière-petite fille» de l'une des reines de France ayant vécu au seizième siècle ${ }^{2}$. Mais de laquelle exactement? Il s'agit soit de Marguerite d'Angoulême (1492-1549), femme du roi de Navarre Henri d'Albret, connue pour ses ouvres littéraires; soit de Marguerite de Valois (1533-1615), célèbre pour ses aventures amoureuses. C'est le gros homme éméché aux pieds de bouc, que rencontre Marguerite au bord de la rivière, qui l'appelle la «radieuse reine Margot»:

I. Ce projet FNS a commencé en 2009 sous la direction du professeur L. Heller. Y participent A. Dobricyn, È. Nadtočij, N. Boyarskaya et Y. Walther.

2. M. Boulgakov, Le Maître et Marguerite, et autres romans, p. 658. 
"Que vois-je? Est-ce bien elle? C'est bien toi, Claudine, veuve infatigable 3 ? Tu es ici, toi aussi?» Et il s'empressait déjà de venir la saluer, mais Marguerite recula et lui répondit d'un ton digne:

«Fiche le camp et va au diable. Je n'ai rien à voir avec ta Claudine. Prends garde à qui tu t’adresses", puis après un instant de réflexion, elle compléta sa réponse par un long chapelet de jurons [...].

«Aïe!» laissa-t-il échapper à mi-voix en tressaillant. «Daignez me pardonner, radieuse reine Margot! J'ai fait erreur sur la personne. C'est la faute au cognac, maudit soit-il!» [...] et débita tout un fatras de phrases russes et françaises mêlées, où il était question à la fois des noces sanglantes de son ami parisien Guessard, de cognac et de sa consternation pour son erreur navrante ${ }^{4}$.

La noce sanglante sur laquelle pérore le gros est celle de Marguerite de Valois et du roi de Navarre, qui eut lieu en 1572 et se termina par un affreux massacre, la nuit de la Saint-Barthélémy. Quant à François Guessard (1814-1882), ce célèbre homme de lettres publia en 1842 les mémoires et les lettres de la reine Margot. Bulgakov connaissait Guessard par l'article du Dictionnaire encyclopédique de Brockhaus et Efron [Brokgauza i Ėfrona ènciklopedičeskij slovar'] concernant Marguerite de Valois, dont on retrouve des extraits dans les cahiers de matériau devant servir pour le roman. Mais qui est Claudine, la «veuve qui ne se désespère pas»? Pour autant que je puisse en juger, le nom de Claudine n'est mentionné dans aucun des nombreux commentaires du roman (y compris dans les éditions françaises que j’ai pu consulter).

La seule piste que j'ai pu trouver concernant Claudine est une référence à une dame d'honneur de la reine Marguerite de Valois, Claudine de La Tour-Turenne (1520-1591), qui, mariée à quinze ans, était déjà veuve à trente-sept. Toutefois, la manière dont elle a pu attirer l'attention de Bulgakov n'est pas claires. Dans les mémoires de la reine Margot, on ne trouve que très peu d'informations sur Claudine. La reine l'appelle selon le nom de son mari Madame de Tournon, et ne la cite que quelques fois. Madame de Tournon avait un lien de parenté avec la reine mère, Catherine de Médicis, et passait pour une femme sage et prudente, au

3. Littéralement, dans le texte original, 'veuve qui ne se désespère pas'. - N. B.

4. M. Boulgakov, Le Maître et Marguerite, et autres romans, p. 650.

5. Cf. le site sur M.A. Bulgakov, http://www.masterandmargarita.eu/fr/02themas/ h21.html. 
mauvais caractère, une mère de famille nombreuse sévère mais attentionnée. Toutefois, Margot ne souffle mot du veuvage de sa dame d'honneur.

Il est tout à fait possible que Bulgakov se soit fait une idée de la cour de France des $\mathrm{XVI}^{\mathrm{e}}$-XVII ${ }^{\mathrm{e}}$ siècles à travers d'autres sources et ne cite l'éditeur Guessard que de façon arbitraire. Mais de quelles sources précisément il se servait, cela reste difficile à établir.

Laissons donc les tentatives de découvrir la mystérieuse Claudine parmi l'entourage de la reine Margot et tournons-nous vers la description de l'envol de Marguerite au bord de la rivière dans la rédaction du Grand chancelier [Velikij kancler] (1931-1934), une version précédente du roman Le Maître et Marguerite. Malheureusement, du chapitre qui nous intéresse, ont été enlevées cinq feuilles de texte en raison, selon V.I. Losev, des actes trop francs de Marguerite vis-à-vis des ennemis de son bien-aimé ${ }^{6}$. Bien entendu, Bulgakov pouvait avoir d'autres raisons. Parmi les scènes détruites, il y en avait au moins deux qui n'étaient pas liées aux critiques de l'Arbat (les ennemies du Maître): la rencontre de l'homme aux pieds de bouc et de Marguerite et la scène avec Claudine, après laquelle elle apparaît à nouveau, plus loin dans la narration, comme "cette même Claudine». Bulgakov a pu écarter ces scènes à cause de leur érotisme trop explicite.

Dans cette version du roman, le gros homme prend Marguerite pour... $\mathrm{Man}^{\prime} \mathrm{ka}^{7}$. Ici la fameuse Man' $\mathrm{ka}^{8}$, tout comme la première femme du Maître (soit Varen'ka, soit Manečka) est une petite-bourgeoise, un personnage passager de l'histoire, incapable d'atteindre l'amour véritable. Pourtant le gros homme, s'étant excusé, ne donne aucune explication liée à Marguerite de Valois et l'appellation la "radieuse reine Margot" n'apparaît pas dans cette rédaction. Le fait est que Marguerite est ici plus proche de la Marguerite de Goethe (Gretchen). Mais même sans être reine, Marguerite a su se présenter à l'assemblée de façon que personne n’a le moindre doute sur la manière dont il faut se comporter à son égard.

Enfin, cette même Claudinette s'approcha de Marguerite et lui demanda d'où elle venait et qui elle était.

6. V.I. Losev, «Kommentarii», p. 962.

7. M.A. Bulgakov, "Moj bednyj, bednyj Master...", p. 169 sq. Nous nous référons ici, ainsi que dans les notes 9 et 23, au texte russe, car plusieurs fragments des premières versions du roman n'ont pas été traduits en français.

8. Prénom typique des personnages féminins dans les feuilletons de Bulgakov. 
"Je suis Marguerite», répondit celle-ci, et elle enfonça son balai dans le sol.

Ces paroles produisirent un effet singulier. [...]

L'on commença à régaler Marguerite. Verrat lui proposa un sandwich au saumon dans lequel il venait tout juste de mordre, ce qui lui valut de la part de Claudinette un coup sur la gueule?

Les fragments conservés suffisent pour se convaincre que Claudine, dont il n'est resté dans la rédaction définitive que le prénom, a probablement été conçue comme une sorcière remplissant les fonctions de dame d'honneur. Le suffixe diminutif caressant -očk-dans le prénom de l'héroïne (Clodinočka - Claudinette) permet de dessiner, au moins de manière générale, son portrait: jeune, attirante et enjouée, elle appelle assurément la sympathie.

Il parait évident que la figure de Claudine a été inventée par Bulgakov avant que Marguerite ne soit associée à la reine française. Il est tout à fait possible que Claudine ait dû apparaître une fois encore dans le roman, au bal qui, dans cette rédaction précoce, avait lieu dans la chambre de Woland. Toutefois, on ne peut que faire des suppositions sur le rôle qu'elle devait jouer ici, dans la mesure où la scène du "petit bal» ${ }^{10}$, comme l'appelaient entre eux Bulgakov et sa femme, fut également supprimée.

Ainsi, qui est donc la Claudine de Bulgakov? Y avait-il dans le contexte artistique proche de Bulgakov une Claudine qu'il eût valu la peine de mentionner dans le roman? Trouve-t-on dans les livres que Bulgakov a lus des personnages appelés Claudine?

La première de ces Claudine est l'héroïne éponyme du cycle scandaleux de romans: Claudine à l'école, Claudine à Paris, Claudine en ménage, Claudine s'en va (Journal d'Annie), La retraite sentimentale (1900-1907), écrits par Sidonie Gabrielle Colette, la plus éclatante des représentantes de la Belle Epoque. Le type de «l'espiègle séductrice» ${ }^{11}$ qu'elle créa permit en son temps à J. Kristeva de comparer ce cycle aux classiques du roman libertin ${ }^{12}$.

9. M.A. Bulgakov, "Moj bednyj, bednyj Master...", p. 169.

Io. B.V. Sokolov, Bulgakovskaja ènciklopedija, p. 139.

II. J. Kristeva, Colette, p. 33.

I2. Ibid., p. 55. 
Le premier roman de Colette, Claudine à l'école, est écrit sous la forme d'un journal d'une écolière de province de classe terminale, un peu sauvage et sans complexes, plus proche du garnement que de la jeune demoiselle. Elle décrit son amour pour une institutrice, et ensuite ses relations, non dénuées d'une nuance sado-masochiste, avec sa camarade petite Luce qui sollicite avec insistance l'amour de Claudine.

Ayant déménagé à Paris (Claudine à Paris), Claudine tombe amoureuse et se marie avec un homme bien plus âgé qu'elle. Dans la tourmente de la vie de bohème parisienne (Claudine en ménage), Claudine noue une relation sentimentale avec une jeune et languissante aristocrate, relation qui se termine peu après que Claudine a rendu son mari jaloux de son amante.

Le quatrième roman, Claudine s'en va (Journal d'Annie), se présente comme le journal d'une amie de Claudine. Annie est une jeune femme silencieuse et renfermée, la dernière véritable épouse, qui, s'étant contre toute attente révoltée, quitte son mari infidèle. Pendant ce temps, Claudine et son mari vieillissant fuient Paris la perfide afin de préserver leur grand amour.

Et enfin, dans le dernier roman, La retraite sentimentale, Claudine mène une vie de recluse en attendant que son mari malade revienne de cure. Lorsque celui-ci revient, Claudine, ne parvenant pas à se faire à sa vieillesse et gardant en mémoire l'image véritable de son bien-aimé, refuse de le recueillir. Et il meurt ainsi rejeté. Toutefois cette perte n'a pas brisé Claudine: absorbée en elle-même, elle vit en pleine harmonie avec la nature, assurant à son amie lors d'une rencontre:

C'était le plus bel amour, celui qui vit de lui-même et demeure après la vie. [...] je n'ai pas perdu mon amour! Croyez ce que je dis - ou bien que ma raison mabandonne un peu, cela ne fait rien... ${ }^{13}$

Ainsi, nous trouvons chez Colette à la fois la jeune et séduisante Claudinette, ainsi que la veuve trentenaire qui ne se désespère pas (rappelons que Marguerite a également trente ans). Malgré une certaine contradiction dans la figure de la veuve ${ }^{14}$, c'est bien à elle qu'appartiennent ces paroles sur l'amour éternel, qui ne pouvaient laisser Bulgakov

13. Colette, Euvres, p. 946.

I4. Le dernier roman sur Claudine, écrit par Colette pendant sa procédure de divorce, reproduit le compromis forcé de sa vie, altérant l'image de Claudine. 
indifférent. Comme d'ailleurs la mystique Claudine, "exaltée et sauvage comme une jeune druidesse ${ }^{15}$, ayant fait de son amour toute une religion, prête à attendre le reste de sa vie une rencontre avec son amant "de l'autre côté de la vie!» ${ }^{16}$.

Les héroïnes de Colette sont en bonne partie autobiographiques. Si en Claudine se reflètent son genre de vie provocateur, son "hermaphrodisme mental ${ }^{17}$ et ses inclinations bisexuelles, en Annie se déverse son aspiration à la révolte contre l'arbitraire du mari. Et en même temps, les deux héroïnes (ainsi que Colette) rêvent au véritable amour: Claudine essaie de le conserver et Annie de le trouver. Rappelons qu'après sa séparation d'avec son tyran de mari, qu'elle aimait malgré le fait qu'il la trompât constamment, Colette trouve asile pour un temps chez la marquise Mathilde de Morny (ou Missy), avant de commencer à se produire au théâtre: danser sur scène et jouer des pantomimes, parfois presque nue dans des scènes ouvertement érotiques. En 1907, la police fut obligée d'interdire le spectacle Rêve d'Egypte ${ }^{18}$ qui passait alors au Moulin Rouge et dans lequel Colette se livrait à un strip-tease et s'abandonnait à l'amour dans les étreintes de Missy, qui jouait le rôle masculin.

Il est tout à fait possible que Bulgakov, qui manifestait un intérêt avide pour les modes de vie étrangers et en particulier pour celui de Paris, ait pu connaître les romans de Colette par sa deuxième épouse L.E. Belozerskaja qui vécut à Paris de 1920 à 1921, c'est-à-dire, juste au moment où, après la sortie du nouveau roman de Colette Chéri (1920), le nom de l'écrivain se trouvait à nouveau sur toutes les lèvres. Il n'est pas exclu que Belozerskaja ait connu Colette comme scandaleuse actricemime et même comme collègue de scène. Le fait est que Belozerskaja, ancienne élève de l'Ecole d'art du ballet, avait un temps travaillé comme danseuse dans le célèbre music-hall parisien des Folies Bergères. La façon toute circonstanciée qui accompagne le dénombrement et la mention des plus éclatantes actrices des années 1920 et qu'elle utilise dans ses mémoires de 1968, après tant d'années, pour décrire «la structure de la grande revue de Paris ${ }^{19}$, trahit son intérêt passé pour l'industrie

\footnotetext{
15. Colette, Euvres, p. 662.

I6. Ibid.

17. J. Kristeva, Colette, p. 25.

I8. H. Dufour, Colette, la vagabonde assise, p. 210-214.

19. L.E. Belozerskaja-Bulgakova, Vospominanija, p. 55.
} 
parisienne du théâtre et du spectacle. Il est vrai qu'alors Colette ne se produit plus sur scène, mais la légende de sa scandaleuse prestation au Moulin Rouge avait sans doute perduré dans le milieu théâtral. Il n'est pas exclu que Bulgakov lui-même ait lu les romans de Colette. Le premier roman sur Claudine avait tout du moins été traduit en russe et publié sous le pseudonyme de son mari (Willy) ${ }^{20}$. Sans parler du fait que Bulgakov, avec sa connaissance du français, pouvait tout aussi bien lire Colette dans l'original.

Un autre prototype de la Claudine de Bulgakov a pu être la soubrette de la pièce de Molière Georges Dandin ou le mari confondu (1668). Durant plusieurs années, Bulgakov écrit La Cabale des dévots (Molière) [Kabala svjatoš (Mol'er)] (1929-1931) pour le Théâtre d'art de Moscou (MXAT), adapte Le bourgeois gentilhomme pour le Théâtre-studio Ju. Zavadskij, répète et monte péniblement des pièces de Molière ou sur Molière. Au printemps 1933, Bulgakov termine la Vie de Monsieur de Molière [Žizn' gospodina de Mol'era]. Et au mois de septembre de la même année, dans une version précoce du Maître et Marguerite, apparaissent deux nouvelles scènes : la non reconnaissance de Marguerite et la rencontre de Claudine avec sa maîtresse.

La Claudine de Molière est un personnage typique de la comédie de mœurs: c'est une servante, belle, intelligente et vive, qui se bat pour sa dignité de femme. Elle est dévouée à sa dépravée maîtresse et, en couvrant les aventures amoureuses de celle-ci, aide à rouler son empoté de mari. Dans la scène du rendez-vous nocturne d'Angélique avec son soupirant Clitandre, ce dernier prend par ailleurs la servante pour sa dame.

Clitandre, à Claudine: Madame.

AngÉLIQue, à Lubin: Quoi?

Lubin, à Angélique: Claudine.

Claudine: Qu'est-ce?

Clitandre, à Claudine: Ah! Madame, que j'ai de joie!

Lubin, à Angélique: Claudine, ma pauvre Claudine.

Claudine, à Clitandre: Doucement, Monsieur.

AngéLIQue, à Lubin: Tout beau, Lubin.

Clitandre: Est-ce toi, Claudine?

Claudine: Oui.

Lubin: Est-ce vous, Madame?

20. K. Willy, Klodina v škole. 
AngéliQue: Oui.

Claudine: Vous avez pris l'une pour l'autre ${ }^{21}$.

Il est vrai que la Claudine de Molière n'est pas encore mariée et ne peut donc être veuve. L'épisode avec Claudine a pu attirer Bulgakov par le mouvement théâtral standardisé de la non reconnaissance (la dimension dramaturgique de la prose de Bulgakov et la théâtralité du Maître et Marguerite ont été passablement étudiées). Le renvoi littéral à Molière est parfaitement légitime. Ainsi dans le dernier chapitre de la rédaction du Roman fantastique [Fantastičeskij roman], le Maître transfiguré apparaît sous les traits de Molière: "En catogan poudré", vêtu du "vieux pourpoint familier, sa canne à la main ${ }^{22}$. C'est pourquoi l'apparition de Claudine dans Le Maître et Marguerite peut aussi être comprise comme un tribut rendu à Molière et au théâtre.

Il ne faut pas oublier que Claudine, chez Bulgakov, ne devient veuve que dans la variante tardive du roman. Avant cela, elle n'est que Claudinette. Pendant ce temps, l'image de Marguerite a pu changer, et il est tout naturel que la figure de Claudine subisse à son tour une certaine évolution. Sans parler du fait que l'idée du sort de la veuve ait pu visiter Bulgakov parce qu'il était atteint de la même maladie dont était mort son père. Comme si les doutes de Bulgakov, l'espoir et la peur avaient trouvé leur reflet dans cette association de mots "la veuve qui ne désespère pas»: soit celle qui vit avec foi et espoir, sans tomber dans le péché, soit la veuve gaie ${ }^{23}$, embourbée dans le péché (la traduction par «veuve infatigable» qu'en donne Françoise Flamand ${ }^{24}$ s'avère plus proche de la deuxième signification, mais elle perd la nuance, importante pour Bulgakov, de désespoir et de tristesse). Il n'est pas anodin que Bulgakov introduise l'«amour éternel, fidèle» dans le texte en 1940, alors qu'il sait que ses jours sont comptés ${ }^{25}$.

Le plus probable est que, tout comme dans la figure de Marguerite se rencontraient plusieurs Marguerite, dans l'image collective de

2I. Molière, Euvres complètes, vol. 2, p. 491 sq.

22. M. Boulgakov, Le Maître et Marguerite, et autres romans, p. 891.

23. Cf. l'amie de Niza, Enante, «la veuve qui ne se désespère pas» dans une version (datant de 1928-1937) du roman de Bulgakov (M.A. Bulgakov, "Moj bednyj, bednyj Master...", p. 603).

24. M. Boulgakov, Le Maître et Marguerite, et autres romans, p. 650.

25. M.O. Čudakova, "Arxiv M.A. Bulgakova», p. 140. 
Claudine se sont recoupées plusieurs Claudine, qui avaient, aux yeux de Bulgakov, beaucoup en commun. Remarquons avant tout que le prénom français de Claudine vient du latin claudus, qui signifie 'boiteux, mutilé, estropié'. Le nom de Claudius fut porté par plusieurs empereurs romains, parmi lesquels Tibère (Tiberius Claudius Nero, 42 av. J.-C. 37 apr. J.-C.). Ce prénom convient très bien à la sorcière: le fait de boiter comme d'autres défauts physiques sont considérés depuis très longtemps comme un moyen de dénoter le coquin. Dans notre cas, l'appartenance de l'héroïne aux forces impures est comme ancrée dans son prénom même. Une autre particularité constante de Claudine est son implication dans des histoires d'amour de toutes sortes: adultères, actes de dépravation, relations homosexuelles, etc. On peut citer à ce propos le roman de l'écrivain belge Camille Lemonnier Claudine Lamour (1893), dans lequel Amour devient le second prénom de la star parisienne Claudine. Bulgakov a pu voir, si ce n'est le roman lui-même, du moins l'article sur son auteur dans l'Encyclopédie littéraire, qu'il possédait chez lui ${ }^{26}$.

D'une façon ou d'une autre, l'image qui s'est finalement constituée de Claudine est tout à fait à l'unisson des leitmotivs du roman bulgakovien : démonisme ou simplement appartenance aux forces impures, connotations érotiques diverses et amour de la liberté. Bien sûr, les questions de généalogie, comme les "questions de sang", sont, d'après Bulgakov, "ce qu'il y a au monde de plus emmêlé» ${ }^{27}$. C'est pourquoi même si je ne suis pas parvenue à résoudre l'énigme de la mystérieuse veuve de Bulgakov, je me console du fait que ces recherches m’ont apporté une nouvelle façon de regarder le roman bulgakovien, à la lumière de la tradition libertine ${ }^{28}$.

Ainsi, comme la Claudine de Colette, Marguerite est une femme forte, dénuée des préjugés de cagots et libérée en amour. Tout cela est propre à Marguerite, sans qu'elle ait besoin de sorcellerie. Avant sa rencontre avec Azazello, Marguerite est décrite comme une femme belle et intelligente «qui avait toujours au fond des yeux une petite flamme

26. A.P. Končakovskij, Biblioteka Mixaila Bulgakova, p. 39.

27. M. Boulgakov, Le Maître et Marguerite, et autres romans, p. 658.

28. D'après Y. Stalloni, le mot «libertin» est affecté, dès le XVII e siècle, de deux sens. Le premier, conformément à l'étymologie (latin libertinus 'esclave affranchi'), renvoie au choix de la liberté et au refus de certaines contraintes institutionnelles, notamment religieuses. A côté de ce libertinage philosophique, on trouve un libertinage de mœurs, un choix de liberté (sexuelle principalement), de licence, de débauche (Y. Stalloni, Dictionnaire du roman, p. 135). 
indéchiffrable", une "sorcière qui louchait imperceptiblement d'un œil» ${ }^{29}$. Cette caractéristique est habituellement utilisée par Bulgakov comme la plus haute appréciation de l'attirance sexuelle d'une jeune femme (cf. Eudoxie de la Garde blanche [Belaja gvardija]) ${ }^{30}$. La sorcière bulgakovienne comme objet érotique joue le même rôle que la femme démoniaque, que la femme fatale.

Marguerite est «intérieurement libre et c'est pour cela qu'elle est "dissolue" " ${ }^{31}$. Elle n'obtient sa libération extérieure qu'une fois devenue sorcière. Au premier regard, il semble que sa liberté de sorcière ait le caractère proprement débauché, insolent et provocateur du mépris des règles socio-morales. Pourtant il apparaît que seule la sorcière amoureuse Marguerite soit capable d'obtenir la libération de son Maître et de rapprocher l'instant du recouvrement de la "liberté suprême " ${ }^{32}$, au-delà des limites de la vie terrestre.

D'une rédaction du roman à l'autre, on retrouve une phrase à propos de la violence exercée sur le Maître et Marguerite et du dernier espoir en la force de l'au-delà dans leur quête de la justice. Devenir sorcière est le seul moyen pour Marguerite de conquérir la liberté dans un pays totalitaire. Mais l'écrivain ne peut être libre dans une telle société, même si, grâce à la force de l'au-delà, il devient inaccessible au pouvoir des possédants. Le Maître ne devient libre que lorsqu'il a atteint les palais du repos éternel. De cette façon, la libération sorcière devient l'équivalent de l'utopie bulgakovienne du repos éternel ${ }^{33}$.

L'émancipation féminine, écrit J. Kristeva, tout comme, à notre avis, la liberté sorcière, ne sont pas possibles sans une «libération de la sexualité de la femme, laquelle est fondamentalement une bisexualité polyphonique: c'est ce que Colette ne cesse de clamer tout au long de sa vie» ${ }^{34}$ et dans ses romans. Comme Claudine, Marguerite possède une part d'«hermaphrodisme mental» 35 . Rappelons que le titre donné à

29. M. Boulgakov, Le Maître et Marguerite, et autres romans, p. 619.

30. Azazello appelle «sorcière" Anne la peste, dont la définition n’a ici aucune connotation érotique, cf. ibid., p. 706.

3I. V.I. Nemcev, Mixail Bulgakov: Stanovlenie romanista, p. 135.

32. Ibid.

33. Sur les liens entre «utopie et utopie de la sexualité», cf. L. Heller, «A la recherche d'un Nouveau Monde Amoureux".

34. J. Kristeva, Colette, p. 25.

35. Ibid. 
Marguerite de «reine radieuse», qui appartenait à la mère de Bulgakov ${ }^{36}$, fait écho à l'appellation de "radieux gaillard " ${ }^{37}$ donnée par Bulgakov à sa seconde épouse. Sa troisième avait pour sobriquet "Lenka - maître d'équipage» ${ }^{38}$. La figure de Marguerite nourrit l'«hermaphrodisme mental» des femmes aimées par Bulgakov.

Marguerite manifeste sa bisexualité et son polyphonisme sensuel, de façon convaincante, dans un extrait conservé de la scène du bal dans la rédaction précoce du Grand chancelier. Dans ce fragment, la relation lesbienne de Marguerite avec une femme anonyme rappelle le couple Claudine et petite Luce chez Colette. Cependant, la ressemblance est purement extérieure, dans la mesure où même les scènes les plus franches chez Colette n'atteignent pas de tels détails physiologiques (dans la rédaction de 1937, l'hérö̈ne découvre le libertinage saphique chez la bonne, Natacha) :

Des grappes de raisin apparurent sur une petite table devant Marguerite, et elle rit aux éclats: le pied de la coupe était un phallus en or. Avec un grand rire, elle le toucha et il s'anima sous sa main. Alors on vint s'asseoir près d'elle de part et d'autre. Un être velu aux yeux brûlants se colla contre son oreille gauche et lui murmura de séduisantes obscénités, un autre, en frac, la serra de près du côté droit et tenta de l'enlacer tendrement par la taille. La gamine s'installa à croupetons devant Marguerite et commença à lui embrasser les genoux ${ }^{39}$.

Bien évidemment, cet épisode, écrit, de l'avis de M.O. Čudakova, dans un esprit "rabelaisien", a un mode d'expression qui n'est pas tout à fait carnavalesque, et on ne peut l'appeler rabelaisien qu'entre guillemets (comme c'est d'ailleurs le cas chez Čudakova ${ }^{40}$ ). En général chez Bulgakov, l'érotisme sorcier est si intimement lié à la tradition libertine de la débauche, que même le sabbat des sorcières devient plus proche des orgies de salon que des festivités païennes.

36. Lors des spectacles du Nouvel An, la mère de Bulgakov jouait le rôle de la reine des neiges.

37. L.E. Belozerskaja-Bulgakova, Vospominanija, p. 222.

38. V.Ja. Lakšin, Golosa i lica, p. 448.

39. M. Boulgakov, Le Maître et Marguerite, et autres romans, p. 861.

40. M.O. Cudakova, "Arxiv M.A. Bulgakova», p. 108. 
La nudité sorcière, exaltée lors du bal par de piquants accessoires, commence à ressembler au costume français du « $\mathrm{Nu}$ au théâtre ${ }^{41}$. A présent, ce ne sont plus seulement des sorcières, mais des «nus» démoniaques. Si dans la scène du sabbat au bord de la rivière Marguerite n'est qu'une sorcière nue, au bal elle joue déjà le rôle d'une dame ${ }^{42}$. La couleur de son costume, ou plutôt de ses gants, dénote, comme cela a été montré dans l'article de M.N. Zolotonosov, le motif de la séduction, lié au topos de Paris ${ }^{43}$.

Dans l'article «Sabbat des sorcières» [«Šabaš ved'm»] du Dictionnaire encyclopédique de Brockhaus et Efron, deux traditions d'interprétation de ce phénomène sont décrites: l'interprétation folklorique et celle de l'Eglise du Moyen-Age. La première est liée aux représentations animistes des phénomènes de la nature, avec les festivités païennes et la croyance en la magie, les devins, les sorcières, etc.; la seconde reflète le refus par l'Eglise catholique chrétienne des rites païens (où les dieux païens sont assimilés à des serviteurs du diable) et la lutte contre les sectes dualistes. Selon l'Eglise médiévale, ce ne sont plus les pitoyables sorcières, mais les représentants de toutes les classes et de tous les rangs, qui constituent la "synagogue du diable»: les hérétiques et les juifs, les "princes temporels et spirituels» ${ }^{44}$.

Bulgakov, s'appuyant en partie sur cet article, renvoie ces deux interprétations dos à dos, conservant à chacune sa place dans le sabbat qui lui correspond. Dans le premier sabbat, le sabbat folklorique, l'envol au bord de la rivière, la baignade et la danse autour du feu sont décrits de façon assez modeste. Dans le second, par le bal printanier de la pleine lune ou bal des cent rois, est représentée, selon les traditions ecclésiastiques chrétiennes, la "synagogue du diable», et sont reproduits les éléments de la messe noire. Il est évident que c'est le bal-sabbat qui intéresse le plus Bulgakov.

A la lumière de l'interprétation du sabbat par l'Eglise, l'on peut regarder le bal chez Satan comme un attroupement de libertins de types variés: libertins-philosophes (hérétiques, anticléricaux, alchimistes,

4I. Cf. M.A. Vološin, «O nagote», p. 23-26.

42. Cela est attesté par sa pose, le culte qui lui est rendu et, enfin, la rude sortie à l'adresse du chat.

43. M.N. Zolotonosov, Slovo i Telo, p. 442.

44. Brokgauza i Ėfrona ènciklopedičeskij slovar', p. 83. 
etc.), débauchés (sorcières, anciennes femmes fatales et grandes amantes, entremetteuses, jouisseuses en tous genres, etc.) et enfin libertins que l'on pourrait qualifier de philosophes-débauchés, qui utilisent la sphère sexuelle comme moyen de s'affranchir et d'atteindre leur objectif.

La Marguerite de Bulgakov et la Claudine de Colette élaborent leur système de libertinage dans les conditions de l'émancipation féminine. Ce sont les "nouvelles Eve» ${ }^{45}$. Toutefois, Marguerite n'est pas une simple femme, c'est aussi une sorcière salvatrice, capable d'obtenir pour l'homme aimé la «liberté suprême». Mais malgré le salut et le tant attendu repos éternel, l'«ancien Adam» aimerait parfois croire que s'il trouvait le repos avant sa femme (-sorcière), celle-ci, à l'instar de la veuve Claudine, garderait alors son "amour fidèle et éternel " dans l'attente d'une rencontre de l'autre côté de la vie.

Natalia BOYARSKAYA

Université de Lausanne

Traduit du russe par Arnaud Nicod-Clément

45. Cf. A. Mag, «Novaja Ėva i prežnij Adam». 


\section{BIBLIOGRAPHIE}

Belozerskaja-Bulgakova, Ljubov' Evgen'evna, Vospominanija, Moskva, Xudožestvennaja literatura, 1989.

Boulgakov, Mikhail (Bulgakov, Mixail Afanas'evič), Le Maître et Marguerite, et autres romans, tr. Françoise Flamant, Jean-Louis Chavarot avec la collaboration de Christian Rouquet et Edith Scherrer, [Paris], Editions Gallimard, 2004.

Bulgakov, Mixail Afanas'evič, "Moj bednyj, bednyj Master...": Polnoe sobranie redakcij i variantov romana "Master i Margarita", Moskva, Vagrius, 2006.

Brokgauza i Ėfrona ènciklopedičeskij slovar', Sankt-Peterburg, Tipografija Akcionernogo Obščestva Brokgauz-Èfron, 1890-1907, vol. XXXIX (77), 1903.

Colette, Sidonie Gabrielle, Euvres, vol. 1, Paris, Editions Gallimard, 1984.

Čudakova, Mariètta Omarovna, "Arxiv M.A. Bulgakova: Materialy dlja tvorčeskoj biografii pisatelja», Zapiski Otdela rukopisej Gosudarstvennoj biblioteki SSSR imeni V.I. Lenina, 37 (1976), p. 25-151.

Dufour, Hortense, Colette, la vagabonde assise, Monaco, Editions du Rocher, 2000.

Heller, Leonid (Geller, Leonid Mixajlovič), "A la recherche d'un Nouveau Monde Amoureux: L'Utopie russe et la sexualité », Revue des études slaves, LXIV/4 (1992), p. 583-602.

Konča kovskij, Anatolij Petrovič, Biblioteka Mixaila Bulgakova. Rekonstrukcija, Kiev, PC World Ukraine, 1997.

Kristeva, Julia, Le génie féminin: la vie, la folie, les mots: Hannah Arendt, Melanie Klein, Colette, vol. 3: Colette, Paris, Fayard, 2002. LAKšın, Vladimir Jakovlevič, Golosa i lica, Moskva, Geleos, 2004. 
Lebedev-Poljanskij (Lebedev), Pavel Ivanovič, Bespalov, Ivan Mixajlovič et al. (otv.red./éds), Literaturnaja ènciklopedija $v 11$ tomax, Moskva, Sovetskaja ènciklopedija, 1932, vol. 6.

Lemonnier, Camille, Claudine Lamour, Paris, Editions Ollendorff, 1903 (1893).

Losev, Viktor Ivanovič, «Kommentarii », in M.A. Bulgakov, "Moj bednyj, bednyj Master... ", p. 935-1006.

MAG, Anneliza, "Novaja Èva i prežnij Adam», Gendernye issledovanija, 12 (2004), p. 72-89.

Molière, Euvres complètes, Paris, Editions Gallimard, 1971, vol. 1-2.

Nemcev, Vladimir Ivanovič, Mixail Bulgakov: stanovlenie romanista, Saratov, Izdatel'stvo Saratovskogo universiteta, 1991.

Sokolov, Boris Vadimovič, Bulgakovskaja ènciklopedija, Moskva, Lokid/ Mif, 1996.

Stalloni, Yves, Dictionnaire du roman, Paris, A. Colin, 2006.

Vološin, Maksimilian Aleksandrovič, "O nagote" (1914), in M.A. Vološin, Sobranie sočinenij v 10 tomax, Moskva, Ėllis Lak, 2007, vol. 5, p. 23-26.

Willi, Kolett, Klodina v škole [Claudine à l'école], tr. L.N. Klod, Moskva, Knigoizdatel'stvo «Osnova», 1908.

Zolotonosov, Mixail Naftal'evič, Slovo i Telo, Moskva, Ladomir, 1999. 
